\title{
Experience Improves Feature Extraction in Drosophila
}

\author{
Yueqing Peng, ${ }^{1,2}$ Wang Xi, ${ }^{1,2}$ Wei Zhang, ${ }^{1,2}$ Ke Zhang, ${ }^{1,2}$ and Aike Guo ${ }^{1,3}$ \\ ${ }^{1}$ Institute of Neuroscience, Key Laboratory for Neurobiology, Shanghai Institutes for Biological Sciences, Chinese Academy of Sciences, Shanghai 200031, \\ China, ${ }^{2}$ Graduate School of the Chinese Academy of Sciences, Beijing 100049, China, and ${ }^{3}$ State Key Laboratory of Brain and Cognitive Science, Institute of \\ Biophysics, Chinese Academy of Sciences, Beijing 100101, China
}

Previous exposure to a pattern in the visual scene can enhance subsequent recognition of that pattern in many species from honeybees to humans. However, whether previous experience with a visual feature of an object, such as color or shape, can also facilitate later recognition of that particular feature from multiple visual features is largely unknown. Visual feature extraction is the ability to select the key component from multiple visual features. Using a visual flight simulator, we designed a novel protocol for visual feature extraction to investigate the effects of previous experience on visual reinforcement learning in Drosophila. We found that, after conditioning with a visual feature of objects among combinatorial shape-color features, wild-type flies exhibited poor ability to extract the correct visual feature. However, the ability for visual feature extraction was greatly enhanced in flies trained previously with that visual feature alone. Moreover, we demonstrated that flies might possess the ability to extract the abstract category of "shape" but not a particular shape. Finally, this experience-dependent feature extraction is absent in flies with defective MBs, one of the central brain structures in Drosophila. Our results indicate that previous experience can enhance visual feature extraction in Drosophila and that MBs are required for this experience-dependent visual cognition.

Key words: learning and memory; Drosophila; mushroom body; visual feature; experience-dependent behavior; flight simulator

\section{Introduction}

Many species can perceive multiple visual features of an object. Fruit flies can also learn to recognize a particular visual feature, such as color, shape, and motion (Quinn et al., 1974; Menne and Spatz, 1977; Desalomon and Spatz, 1983; Ernst and Heisenberg, 1999; Srinivasan et al., 1999; Tang and Guo, 2001). In Drosophila, the memory traces of two visual features (elevation and contour orientation of the object) are localized in two groups of neurons in the fan-shaped body of the central complex (Liu et al., 2006). Furthermore, the memory for color and shape cues can be retrieved separately after compound conditioning with objects exhibiting both features (Tang and Guo, 2001). In addition, visual perception can also be modulated by previous experience in both vertebrates and invertebrates. Training monkeys to track targets of a particular color leads to a selectivity in the responses of frontal eye field neurons toward stimuli exhibiting that color (Bichot et al., 1996). In honeybees, previous experience also enhances visual pattern discrimination for differently shaped and camouflaged figures (Zhang and Srinivasan, 1994). In the present study, we investigated whether flies can be conditioned to recognize a particular visual feature from objects with other distraction features and whether previous experience with a particular feature

Received 0ct. 16, 2006; revised March 26, 2007; accepted March 27, 2007.

This work was supported by National Science Foundation of China Grants 30270341, 30630028, and 30621004; the Multidisciplinary Research Program (Brain and Mind) of the Chinese Academy of Sciences; National Basic Research Program of China Grants G20000 77800, 2006CB806600, and 2006CB911003; and Knowledge Innovation Engineering Project of the Chinese Academy of Sciences Grants KJCX1-09-03 and KSCX2-YW-R-28. We thank M. Heisenberg for his constructive discussion and M.-M. Poo for a critical reading of this manuscript.

Correspondence should be addressed to Aike Guo at the above address. E-mail: akguo@ion.ac.cn.

DOI:10.1523/JNEUROSCI.0472-07.2007

Copyright $\odot 2007$ Society for Neuroscience $\quad$ 0270-6474/07/275139-07\$15.00/0 helps subsequent recognition of that feature among combinatorial features.

The mushroom bodies (MBs) constitute an important brain structure in insects (for reviews, see Heisenberg, 1998; Zars, 2000; Heisenberg, 2003), and are essential for olfactory learning and memory in Drosophila (Heisenberg et al., 1985; De Belle and Heisenberg, 1994; Connolly et al., 1996). Studies have shown that MBs are also required for some visual cognitive processes, such as context generalization (Liu et al., 1999) and choice behavior facing conflicting cues (Tang and Guo, 2001). Recent reports have shown that MBs can also regulate sleep in Drosophila (Joiner et al., 2006; Pitman et al., 2006). Here, we examined whether Drosophila MBs are also involved in experience-related visual feature extraction among combinatorial features.

In the present study, by using a flight simulator, we demonstrated that previous experience enhances shape or color feature extraction among objects with combinatorial shape-color features in Drosophila. In addition, Drosophila MBs are required for this experience-dependent visual feature extraction. Our findings indicate that the Drosophila brain can use previous experience to improve certain visual cognitive abilities.

\section{Materials and Methods}

Drosophila strains. Flies were cultured on standard medium as described previously (Guo et al., 1996) at $25^{\circ} \mathrm{C}$ and $60 \%$ relative humidity with a $12 \mathrm{~h}$ light/dark cycle. Wild-type Berlin (WTB) and mutant MB miniature $^{1}\left(\mathrm{mbm}^{1}\right)$ flies with a WTB genetic background were used. Hydroxyurea (HU)-treated flies were obtained by following the previous procedure (De Belle and Heisenberg, 1994) with WTB. CantonS (CS) flies also served as an additional wild type. Female flies (3-4 d old) were briefly immobilized by cold anesthesia and then were glued (Locktite UV glass glue) by their heads and thoraxes to triangle-shaped hooks $(0.05 \mathrm{~mm}$ in 
diameter) the day before the experiment. Flies were then kept individually in small chambers and were fed with a sucrose solution overnight.

Apparatus. The device was a flight simulator that was originally built by Reichardt and Wenking (1969) and improved by Heisenberg and Wolf (1979, 1988) (Wolf and Heisenberg, 1991). The flies with hooks were fixed to a torque meter (Goetz, 1964) in the center of a circular panorama (44 $\mathrm{mm}$ diameter) that could be rotated by a fast electric motor. The torque meter measured each fly's angular momentum around its vertical body axis and rotated the panorama around the fly by a negative feedback mechanism. This arrangement allowed the tethered flies to stabilize and choose their flight orientation with respect to the panorama by adjusting their yaw torque. Flight orientation (panorama position) was recorded continuously and stored in the computer (sampling frequency, $20 \mathrm{~Hz}$ ) for additional analysis. The panorama was divided into four quadrants with the visual objects at their respective centers. An infrared laser beam was switched on when the flight orientation was in the punished quadrants and switched off in the safe quadrants. For conditioning, the object in one quadrant was symmetrically grouped with the object in the opposite quadrant.

Visual stimulus. Upright Ts or inverted Ts measured $40^{\circ}$ vertically and $40^{\circ}$ horizontally. The bars of Ts were $12^{\circ}$ wide. Horizontal bars were $12^{\circ}$ long and $40^{\circ}$ wide. The colored Ts or horizontal bars were printed on ink jet transparency films with a color printer (Epson Photo 925; Epson, Long Beach, CA). Visual stimuli were presented to flies in a dark background. The luminance of different colored objects was calibrated with a luminance meter (LS-100; Minolta, Osaka, Japan).

Data analysis. The fly's performance was evaluated quantitatively over 2 min blocks using the "preference index" (PI), which is defined as follows: $\mathrm{PI}=\left(t_{1}-t_{2}\right) /\left(t_{1}+t_{2}\right)$, where $t_{1}$ is the time the flies spent heading toward the safe quadrants (two opposite quadrants), whereas $t_{2}$ is the time heading toward the quadrants with the punishment (other opposite quadrants). PIs were averaged for further statistics in the test period. One-way $t$ test was used for every comparison $\left({ }^{* * *} p<0.001 ;{ }^{* *} p<0.01 ;{ }^{\star} p<0.05\right.$; two-sided $p$ value). Error bars in the figures are SEMs.

\section{Results}

\section{Experience-dependent shape feature extraction}

As shown in Figure $1 \mathrm{~A}$ (left), there were four different objects. The objects were groups based on the different visual features. Horizontally, the shape feature was the main distinguishing component of the groups. Vertically, the color feature was the main distinguishing component. The ability to select this key component from multiple features is considered as visual feature extraction. To study a fly's ability to perform visual feature extraction, we designed a novel protocol for training the fly to extract either color or shape features among objects with combinatorial colorshape features in a flight simulator (see Materials and Methods). The panorama of the simulator was divided into four quadrants, with visual objects in the dark background located at the center of the quadrants. For shape feature extraction, the identical T patterns (upright or inverted Ts) with different colors were symmet-
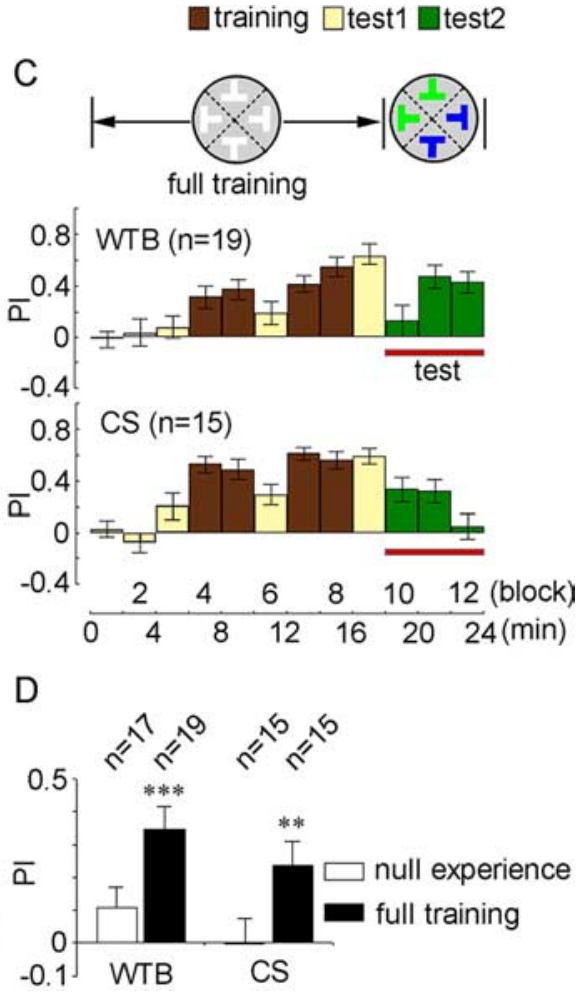

Figure 1. Experience-dependent shape feature extraction in Drosophila. $\boldsymbol{A}$, The paradigm of visual feature extraction. Left, Feature grouping for four differently colored T patterns. The shape feature was the main component to distinguish the difference (solid line boxes), whereas the color feature was the main component to distinguish the vertical difference

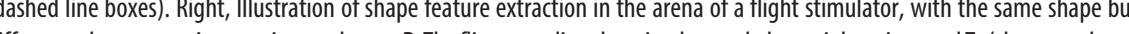
(blocks 9-12) to extract the shape feature from objects with combinatorial shape-color features. C, The flies were fully -type flies recognized the shape feature (blocks 10-12) after previous training. $\boldsymbol{D}$, Statistical analysis demonstrated that flies in each case. Red horizontal lines in this figure and the following figures indicate the Pls in the test period, which were averaged for statistical analysis.

rically arranged in the opposite quadrants (Fig. $1 \mathrm{~A}$, right). Then, the flies were directly conditioned to avoid upright or inverted Ts by punishment with an infrared laser beam. We found that WTB flies showed poor performance in learning to follow the shape feature (Fig. $1 B$ ), with preference indices (PIs) close to zero (Fig. $1 D)$. A similar poor performance was also observed in CS wildtype flies (Fig. $1 B, D$ ). In sharp contrast, when these flies were conditioned to recognize objects with only a single shape feature (white upright or inverted Ts), they exhibited excellent abilities in shape recognition (with high PI in block nine) (Fig. 1C). Thus, wild-type flies exhibited a generally poor ability to learn to extract the shape feature of an object when the shape feature was presented with distraction features, such as color.

To examine whether previous experience can improve visual feature extraction in Drosophila, we first conditioned the flies to learn the shape feature alone (for four blocks) and then tested their ability to recognize that trained shape feature among objects with combinatorial color-shape features (Fig. 1C). As described above, the flies were able to track the object with the conditioned shape (block nine), and the addition of the color feature during the test period did not interfere with the ability to extract the shape feature (blocks 10-12) in both WTB and CS flies (Fig. $1 C, D)$. These results imply that previous training with shape fea- 


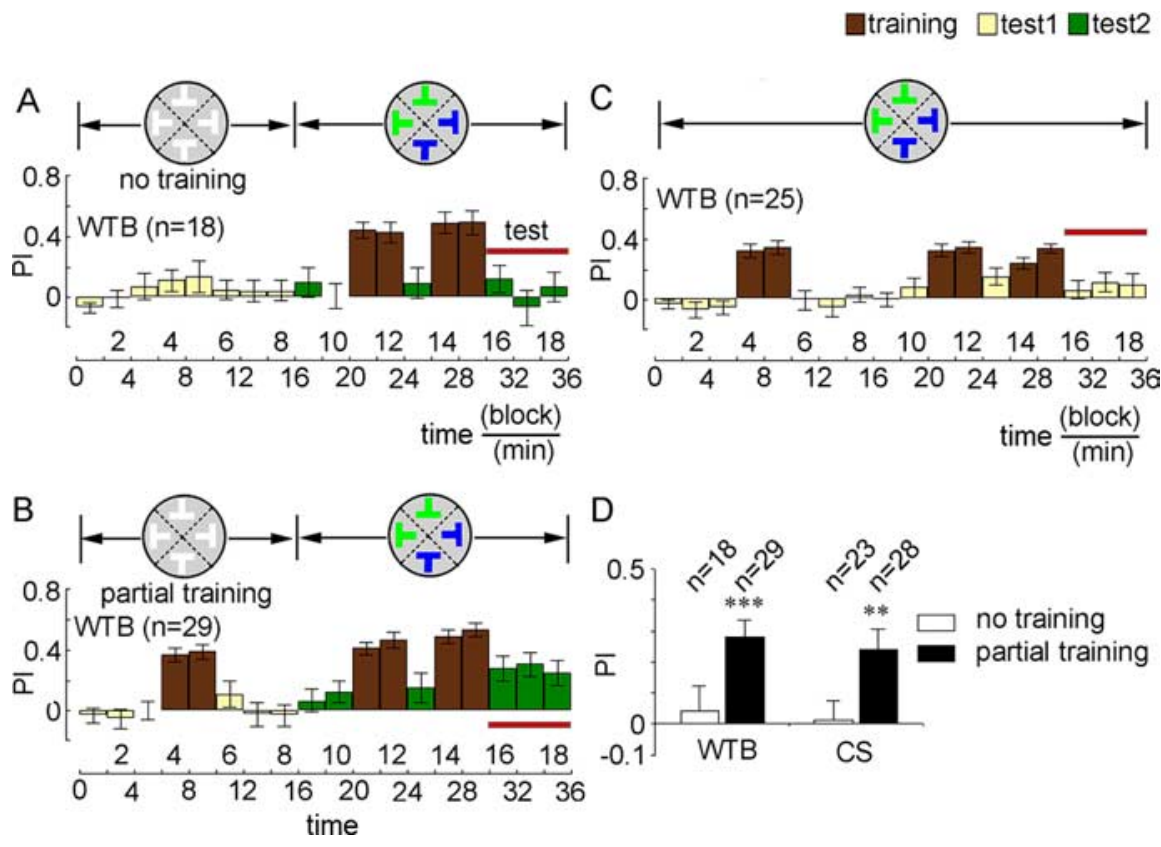

Figure 2. Partial training enhanced shape features extraction. $\boldsymbol{A}$, Previous exposure to the shape feature without training had no effect on the extraction of shape feature in WTB flies. $\boldsymbol{B}$, Partial training with the shape feature alone improved subsequent shape feature extraction in WTB flies (blocks 16-18), although this training did not result in significant memory for the shape feature (blocks 6 - 8). C, WTB flies exhibited poor shape feature extraction after six blocks of conditioning with the shape feature among objects with combinatorial shape-color features. $\boldsymbol{D}$, Compared with previous exposure without training, partial training significantly enhanced the shape feature extraction ability in both WTB and CS flies. Top right corner: brown, training periods; yellow, test periods; green, test periods with new objects.

ture alone could subsequently improve shape feature extraction from objects with combinatorial color-shape features.

Different levels of previous experience may have different effects on visual perception in animals. In honeybees, increasing experience with longer training trials results in greater generalization of visual feature assemblies (Stach and Giurfa, 2005). To further investigate the effect of previous experience on visual reinforcement learning and memory in the visual feature extraction paradigm, we introduced two other levels of previous experience to the flies. Prior exposure of WTB flies to the shape feature alone without conditioning had no effect on shape feature extraction after conditioning with the shape feature among objects with combinatorial shape-color features (Fig. 2A). However, previous "partial" training with the shape feature alone (blocks four to five) (Fig. $2 \mathrm{~B}$ ), which was insufficient for inducing significant memory for the shape feature (blocks six to eight), led to significant PIs in shape feature extraction after subsequent reconditioning with the shape feature among objects with combinatorial shape-color features (blocks 16-18) (Fig. 2B). To exclude the possibility that blocks four and five during the course of partial training had prolonged the duration of conditioning (six blocks, compared with four blocks) (Fig. $1 \mathrm{~B}$ ), perhaps leading to significant PIs during the test period, we designed a 36 min protocol without previous experience to train the flies to extract the shape feature from objects with combinatorial shape-color features. Therefore, compared with the protocol in Figure $1 B$, an additional training of two blocks was introduced in this control experiment. As shown in Figure 2C, wild-type flies (WTB) also showed poor performance in shape feature extraction after six blocks of training. Statistical analysis revealed that partial training significantly improved shape feature extraction among combinatorial shape-color features in WTB flies (Fig. 2D). Similar results were also found in CS wild-type flies. Considering the above experiments (Figs. 1, 2) on shape feature extraction, under conditions of no previous experience with the shape feature ("null experience"), exposure to the shape feature without training ("no training"), as well as "partial training" (two blocks) and "full training" (four blocks) with the shape feature, all of these results suggest that previous experience with a particular visual feature helps to enhance the fly's ability to extract that feature from objects with multiple visual features.

To further explore whether the flies are capable of extracting the abstract category of "shape," but not the particular " $T$ " shape, we performed the following experiment. First, the flies were partially trained to avoid upright/inverted Ts in the shapealone paradigm, and then they were reverse conditioned to avoid inverted/upright Ts in the combinatorial shape-color paradigm. If the flies possessed abstract abilities in visual feature extraction, we would expect good performance during the test period. In contrast, if the flies remembered only the particular $\mathrm{T}$ shape, we would expect poor performance during the test period, because the shape in the second training period is different from the previous trained shape. We found that WTB flies showed a good ability to track the correct shape feature, after the reversal training (Fig. $3 \mathrm{~A}, \mathrm{C}$ ). A similar result was also observed in CS flies (Fig. 3C). As a control, we also partially trained the flies with the color feature (blue/green horizontal bars with identical height) instead of the shape feature in the pretraining period. Consequently, both WTB and CS flies showed poor performance in extracting the shape feature from the combinatorial shape-color features during the test period (Fig. $3 B, C$ ). Together, these results imply that flies possess the ability to extract the abstract category of shape, based on previous experience with that particular shape feature.

\section{MBs are required for experience-dependent shape feature extraction.}

MBs are essential for some visual cognitive behaviors, such as context generalization (Liu et al., 1999) and choice behavior (Tang and Guo, 2001). To investigate MB function in this experience-dependent visual feature extraction, the mutant flies with miniature $\mathrm{MBs}\left(\mathrm{mbm}^{l}\right)$ and flies with $\mathrm{MB}$ ablation by $\mathrm{HU}$ were used. As shown in Figure $4 A$, the effect of previous partial training with the shape feature alone on the subsequent shape feature extraction from the combinatorial shape-color features was completely abolished in HU-treated flies (blocks 16-18). Furthermore, the MB-deficient ( $\mathrm{mbm}^{1}$ and HU-treated) flies still showed poor performance on the shape feature extraction (blocks 10-12), even after full training with the shape feature alone (Fig. 4B). Statistical analysis demonstrated that the PIs in both cases (partial and full training) were not significantly different from zero for both MB-deficient flies (Fig. 4C). Consistent with the finding that MBs are dispensable in Drosophila visual and motor learning (Wolf et al., 1998), we also found that shape 
$\square$ training $\square$ test1 $\square$ test2

A

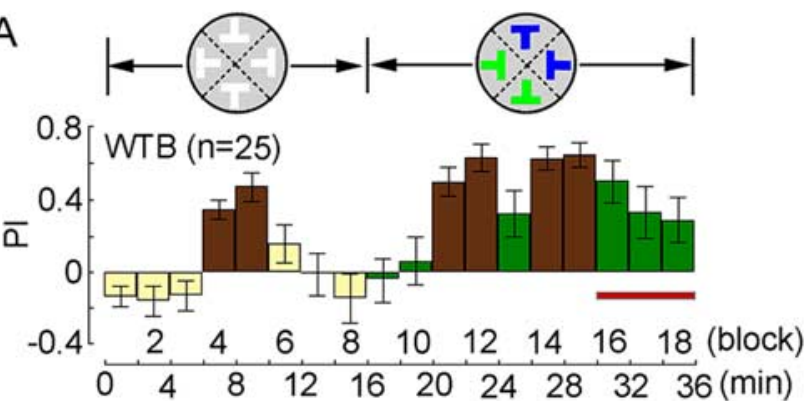

B
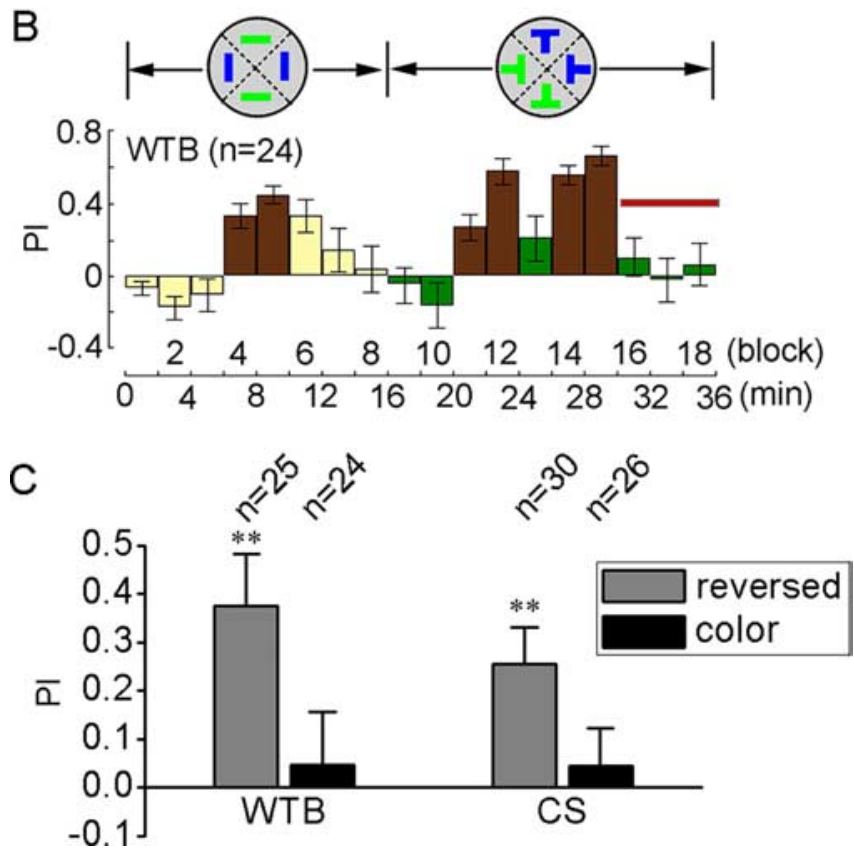

Figure 3. Previous experience with the shape feature but not a particular $T$ shape enhances shape feature extraction from combinatorial shape-color features. A, WTB flies were partially trained with upright/inverted Ts in the shape-alone paradigm, and then they were reversetrained with inverted/upright Ts in the combinatorial shape-color paradigm. The flies showed good shape feature extraction during the test period (marked with the red line). $\boldsymbol{B}$, WTB flies showed poor shape feature extraction after partially trained with green/blue color bars of identical heights. C, Statistical analysis revealed that previous experience with "reversed" training Ts, but not green/blue "color" horizontal bars, could improve shape feature extraction. Top right corner: brown, training periods; yellow, test periods; green, test periods with new objects.

recognition ability in the flight simulator was not affected in either HU-treated or $\mathrm{mbm}^{1}$ flies (block 9) (Fig. 4B). In addition, similar to the wild-type flies (Fig. $1 B$ ), the MB-deficient flies also showed poor shape feature extraction performance in the absence of any previous experience with the shape feature (null experience) (Fig. 4C). These results indicate that MBs are required for experience-dependent shape feature extraction in Drosophila.

Previous studies in the flight simulator reported that the MBs were essential for context generalization in Drosophila (Liu et al., 1999). To exclude the possibility that a simple color change of objects could disrupt recognition of the shape feature in MBdeficient flies, two tasks were designed, including the addition of a uniform color and two consistent colors to the objects after shape conditioning. We found that when a uniform color (green or blue) was added to all objects, MB-deficient flies were capable of recognizing previously conditioned shape feature in the test period, similar to the wild- type flies (Fig. $5 A, B$ ). Furthermore, when the same color was added to the objects with the same shape in a consistent manner, both MB-deficient flies as well as wild-type flies still exhibited recognition of the previous shape feature during the test period (Fig. 5C,D). Thus, the disruption of MB function affected the fly's ability to extract the shape feature only in the presence of a color feature that was inconsistent with the shape feature. This further suggests that the function of the MBs is not for simple feature recognition but for feature extraction from objects with complex and confusing features.

\section{Experience-dependent color feature extraction}

In the last experiment, flies were required to extract the color feature from objects with combinatorial shape-color features. For those flies without previous experience of the color feature (null experience group), both wild-type and MB-deficient $\left(\mathrm{mbm}^{1}\right.$ and HU-treated) flies exhibited poor color feature-extraction performance after direct conditioning with the color feature among objects with the combinatorial shape-color features (Fig. 6A). Subsequently, three different levels of previous experience with the color feature were introduced to flies. We found that previous exposure to the color feature (green/blue horizontal bars) without training slightly improved the color feature extraction ability in wild-type flies but not in MB-deficient flies (Fig. 6B). This color feature extraction ability was further strengthened in wildtype flies exposed to partial and full previous training with the color feature alone, whereas MB-deficient flies remained incapable of performing the task (Fig. 6C,D). Taken with the above findings on shape feature extraction, previous experience with a particular visual feature enhances extraction of that feature from the multiple combinatorial features, and the MBs are required for this experience-dependent visual feature extraction in Drosophila.

\section{Discussion}

In the present study, using novel paradigms in the flight simulator, we demonstrated that previous experience can enhance visual feature extraction in Drosophila, and MBs play an important role in this experience-dependent visual feature extraction. Although flies can be trained to recognize either the shape or color features of an object independently (Tang and Guo, 2001), in the present study, they exhibited a poor ability to directly extract either shape or color features of objects with combinatorial colorshape features (Figs. $1 B, 5 A$ ). This poor performance may result from the interference of other features. When the flies were trained to extract the shape feature, such as upright Ts, coupled with punishment, the association of the color feature with the punishment also occurred simultaneously. During the test period, the color feature would thus interfere with the fly's ability to track the shape feature, leading to low PI scores. Prior experience with a particular feature appears to have left an imprint in the fly's brain that facilitates subsequent training for the extraction of that feature. In humans, the ability to detect or identify objects that have been recently encountered, a phenomenon known as priming, has been intensively studied (for review, see Milner et al., 1998). This imprint effect may function as a primer. Therefore, even partial training can enhance shape or color feature extraction from combinatorial shape-color features in Drosophila.

To further examine a fly's ability to learn objects with combinatorial shape-color features, we tried to design an even more demanding task, in which flies were trained to learn the combi- 
A

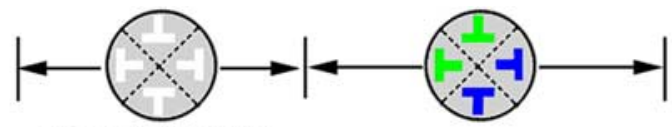

partial training

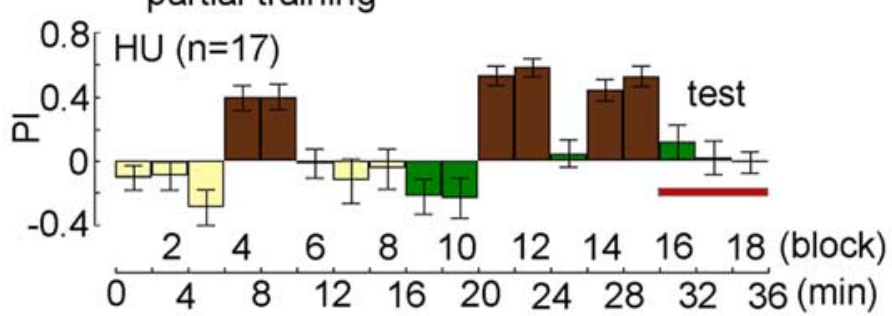

B

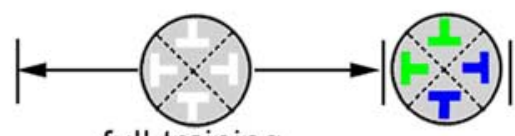

full training
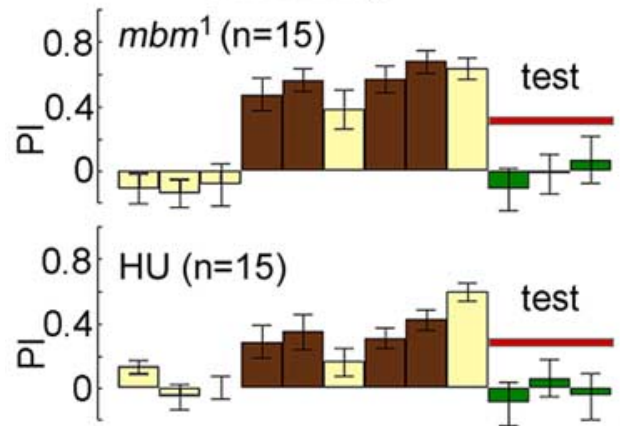

\begin{tabular}{cccccccc} 
& 2 & 4 & 6 & 8 & 10 & 12 (block) \\
\hline 0 & 4 & 8 & 12 & 16 & 20 & 24 (min)
\end{tabular}

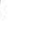

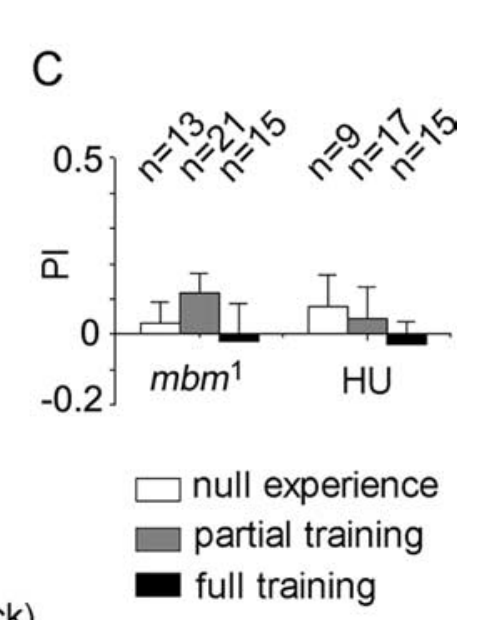

$\square$ null experience

partial training

full training

Figure 4. MBs are required for experience-dependent shape feature extraction in Drosophila. A, Previous partial training with the shape feature alone has no effect on shape feature extraction in $\mathrm{HU}$-treated flies. Brown, training periods; yellow, test periods; green, test periods with new objects. $B$, Both MB-deficient ( $\mathrm{mbm}^{1}$ and $\mathrm{HU}$-treated) flies also showed poor shape feature extraction (blocks $10-12$ ) after full training with the shape feature alone. Note that MB-deficient flies showed learning for shape feature alone (block 9). C, Without (null) or with (partial and full) previous experience of the shape feature, MB-deficient flies exhibited poor performance on shape feature extraction among objects with combinatorial shape-color features, as indicated by PIs close to zero $(p>0.05)$.

nation of the shape and color features, instead of one particular shape or color feature. For example, only the green upright $\mathrm{T}$ is dangerous, whereas the green reverted $\mathrm{T}$ or blue upright $\mathrm{T}$ is safe. We found that flies still exhibited poor performance in this feature-combined learning task (data not shown). As another sample of complex training with combinatorial shape-color features, Brembs and Wiener (2006) have shown that using color as background rather than as a property of the visual object, flies can successfully be trained in a biconditional task in which one pattern (e.g., upright T) is safe in a blue background but dangerous in a green background, whereas the other pattern (e.g., reverted $\mathrm{T}$ ) is safe in a green background and dangerous in a blue background. In addition to training protocols (11 min massed training vs 12 min spaced training), the main difference between the previous and present studies is the method of color stimuli presentation. We used color stimuli as visual features of objects, whereas Brembs and Wiener (2006) used color stimuli as the background. As mentioned above, MBs are required for visual pattern recognition after the environmental conditions are changed (Liu et al., 1999). Our results showed that MB-deficient flies remain capable of recognizing previously trained features when new information is consistently added to the objects but

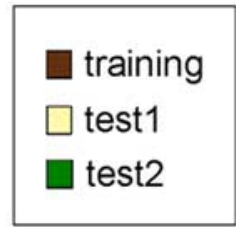

not to the background (Fig. 5). This finding suggests that MBs might not be necessary for basic object generalization when the properties of an object are not altered extensively. One possible reason for different results of $\mathrm{MB}$ function in visual generalization may be because of different experimental conditions, such as the spectral properties of the color stimuli. A recent report shows that the spectral properties of the color stimuli are critical for visual generalization and discrimination learning (Brembs and Hempel de lbarra, 2006). In the present study, the chromaticity of the color stimuli is broad and the luminance is adjusted to the human eye rather than to that of the fly (Minolta LS-100 luminance meter). Another possible reason for different functions of the MBs in these two generalization tasks is that neural mechanisms underlying information processing for the visual object and background might be different, which is also consistent with the different results of feature-combined learning paradigms between our study and a previous study (Brembs and Hempel de lbarra, 2006; Brembs and Wiener, 2006).

In the present study, we found that the flies are capable of learning to recognize the shape feature from combinatorial shape-color features even when the particular feature value are reverse conditioned (Fig. 3). This result implies that the flies may possess the ability to extract the abstract category of shape, beyond a particular shape. Another previous study in the flight simulator showed that visual pattern recognition in Drosophila is translation invariant in the retinal position (Tang et al., 2004). Our results indicate that flies can further distinguish the invariant feature from objects with multiple features. Thus, from the basic pattern recognition to the abstract feature extraction, Drosophila exhibits good performance at various levels of visual cognition. The circuit mechanism underlying the abstract shape feature extraction should be further explored.

Previous studies reported that MB lesions led to an increase in general motor activity in both crickets (Huber, 1955) and fruit flies (Martin et al., 1998). Considering the function of MBs in experience-dependent feature extraction and other visual cognitive behavior (Liu et al., 1999; Tang and Guo, 2001), we propose that MBs are the brain center for comparing previous experience and incoming sensory inputs and for implementing a "gating" mechanism that inhibits unrelated or alternative information while amplifying experience-related signals of the relevant input. In addition to the MBs, this experiencedependent visual feature extraction may also require other brain regions, such as the central complex. However, it is difficult to determine the importance of the central complex for experience-dependent visual feature extraction, because its impairment directly influences visual pattern memory in Drosophila (Liu et al., 2006). 
Previous experience is essential for regulating current and future behaviors in various species (Hofmann and Stevenson, 2000; Levine et al., 2002; Hebda-Bauer et al., 2005). In addition, recent studies have shown a growing interest in discovering cognitive behaviors in fruit flies and its relatives (Greenspan and Van Swinderen, 2004). For example, honeybees possess the ability to perceive symmetrical objects (Giurfa et al., 1996) and to discriminate the abstract concepts of "sameness" and "difference" (Giurfa et al., 2001). We are interested in the combination of these two fields of studies together: previous experience and cognitive behaviors in insects. Our findings demonstrated that even a simple visual cognitive process, such as visual feature extraction, is highly dependent on previous visual experience. Armed with the powerful genetic manipulations of Drosophila, we believe that studying the neural circuit mechanisms underlying such experience-dependent visual processes in Drosophila may contribute to our understanding of high-level cognitive behaviors in general.

\section{References}

Bichot NP, Schall JD, Thompson KG (1996) Visual feature selectivity in frontal eye fields induced by experience in mature macaques. $\mathrm{Na}-$ ture 381:697-699.

Brembs B, Hempel de Ibarra N (2006) Different parameters support generalization and discrimination learning in Drosophila at the flight simulator. Learn Mem 13:629-637.

Brembs B, Wiener J (2006) Context and occasion setting in Drosophila visual learning. Learn Mem 13:618-628.

Connolly JB, Roberts LJ, Armstrong JD, Kaiser K, Forte M, Tully T, Kane CJ (1996) Associative learning disrupted by impaired Gs signaling in Drosophila mushroom bodies. Science 274:2104-2107.

De Belle JS, Heisenberg M (1994) Associative odor learning in Drosophila abolished by chemical ablation of mushroom bodies. Science 263:692-695.

Desalomon CH, Spatz HC (1983) Color-vision in Drosophila melanogaster: wavelength discrimination. J Comp Physiol 150:31-37.

Ernst R, Heisenberg M (1999) The memory template in Drosophila pattern vision at the flight simulator. Vision Res 39:3920-3933.

Giurfa M, Eichmann B, Menzel R (1996) Symmetry perception in an insect. Nature 382:458-461.

Giurfa M, Zhang S, Jenett A, Menzel R, Srinivasan MV (2001) The concepts of 'sameness' and 'difference' in an insect. Nature 410:930-933.

Goetz KG (1964) Optomotorische Untersuchung des visuellen systems einiger Augenmutanten der Fruchtfliege Drosophila. Kybernetik 2:77-92.

Greenspan RJ, Van Swinderen B (2004) Cognitive consonance: complex brain functions in the fruit fly and its relatives. Trends Neurosci 27:707-711.

Guo A, Li L, Xia SZ, Feng CH, Wolf R, Heisenberg M (1996) Conditioned visual flight orientation in Drosophila: dependence on age, practice, and diet. Learn Mem 3:49-59.

Hebda-Bauer EK, Watson SJ, Akil H (2005) Cognitive performance is highly sensitive to prior experience in mice with a learning and memory deficit: failure leads to more failure. Learn Mem 12:461-471. $\square$ training $\square$ test1 $\square$ test2

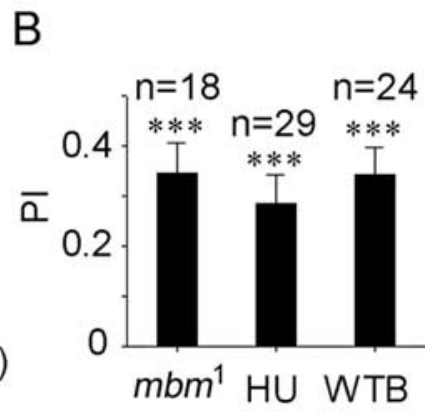

Figure 5. MBs are dispensable for simple shape feature recognition from consistent combinatorial features. $A, H U$-treated flies

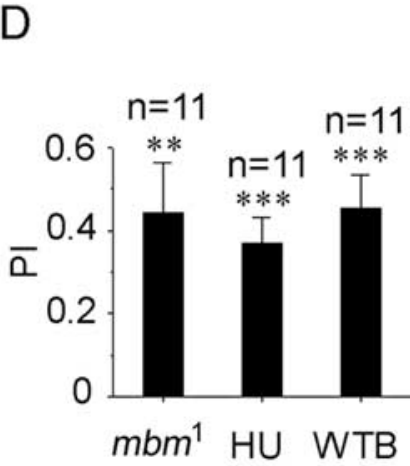
(block) (blocks $10-12$ ) when a uniform color (green or blue) was added to the objects. Note that only rognition in the task, as shown in $A . C$ When trained with shape features alone, HU-treated flies also recognized 列 periods; yellow, test periods; green, test periods with new objects.

Heisenberg M (1998) What do the mushroom bodies do for the insect brain? An introduction. Learn Mem 5:1-10.

Heisenberg M (2003) Mushroom body memoir: from maps to models. Nat Rev Neurosci 4:266-275.

Heisenberg M, Wolf R (1979) On the fine structure of yaw torque in visual flight orientation of Drosophila melanogaster. J Comp Physiol 130:113-130.

Heisenberg M, Wolf R (1988) Reafferent control of optomotor yaw torque in Drosophila melanogaster. J Comp Physiol A Neuroethol Sens Neural Behav Physiol 163:373-388.

Heisenberg M, Borst A, Wagner S, Byers D (1985) Drosophila mushroom body mutants are deficient in olfactory learning. J Neurogenet 2:1-30.

Hofmann HA, Stevenson PA (2000) Flight restores fight in crickets. Nature 403:613.

Huber F (1955) Sitz und bedeutung nervöser Zentren für Instinkthandlungen beim Mannchen von Gryllus campestris L. Z. Tierpsychol 12:12-48.

Joiner WJ, Crocker A, White BH, Sehgal A (2006) Sleep in Drosophila is regulated by adult mushroom bodies. Nature 441:757-760.

Levine JD, Funes P, Dowse HB, Hall JC (2002) Resetting the circadian clock by social experience in Drosophila melanogaster. Science 298:2010-2012.

Liu G, Seiler H, Wen A, Zars T, Ito K, Wolf R, Heisenberg M, Liu L (2006) Distinct memory traces for two visual features in the Drosophila brain. Nature 439:551-556.

Liu L, Wolf R, Ernst R, Heisenberg M (1999) Context generalization in Drosophila visual learning requires the mushroom bodies. Nature 400:753-756.

Martin JR, Ernst R, Heisenberg M (1998) Mushroom bodies suppress locomotor activity in Drosophila melanogaster. Learn Mem 5:179-191. 


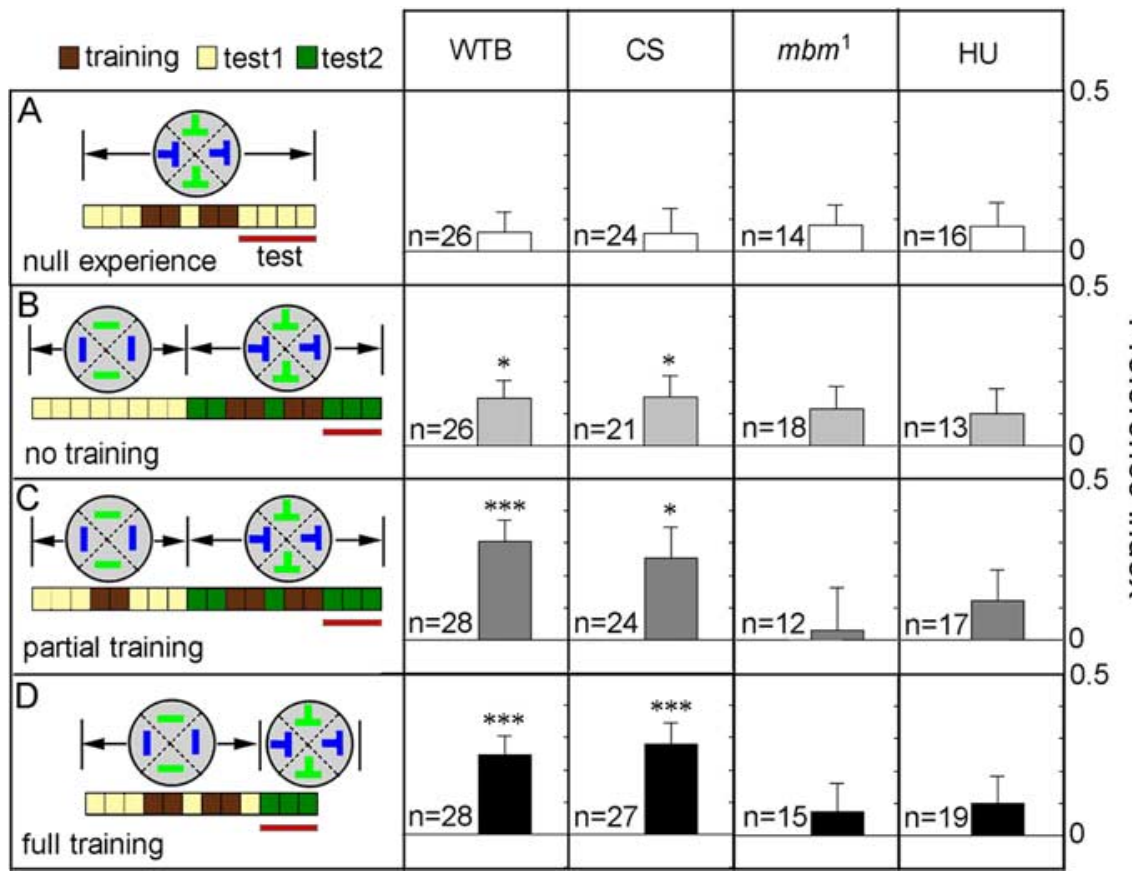

Figure 6. Experience-dependent color feature extraction in Drosophila. $\boldsymbol{A}$, The visual stimuli included four objects with the same color feature but different shape feature in opposite quadrants. All flies without previous experience exhibited poor color feature extraction ( $p>0.05$ ). $\boldsymbol{B}$, The wild-type flies (WTB and CS) exhibited slightly positive preference indices (PIs) for color feature extraction after previous exposure to the color feature without training ( $16 \mathrm{~min}$; green/blue bars). The PIs in MB-deficient $\left(\mathrm{mbm}^{1}\right.$ and $\mathrm{HU}$-treated) flies were not significantly different from zero. C, Partial training with the color feature alone further improved color feature extraction in wild-type flies but not in MB-deficient flies. $\boldsymbol{D}$, Full training also had positive effects on color feature extraction in wild-type flies, but not in MB-deficient flies. Red horizontal lines depict the PIs shown in the figures. Top left corner: brown, training periods; yellow, test periods; green, test periods with new objects.

Menne D, Spatz HC (1977) Color-vision in Drosophila melanogaster. J Comp Physiol 114:301-312.

Milner B, Squire LR, Kandel ER (1998) Cognitive neuroscience and the study of memory. Neuron 20:445-468.

Pitman JL, McGill JJ, Keegan KP, Allada R (2006) A dynamic role for the mushroom bodies in promoting sleep in Drosophila. Nature 441:753-756.

Quinn WG, Harris WA, Benzer S (1974) Conditioned behavior in Drosophila melanogaster. Proc Natl Acad Sci USA 71:708-712.
Reichardt W, Wenking H (1969) Optical detection and fixation of objects by fixed flying flies Naturwissenschaften 56:424-425.

Srinivasan MV, Poteser M, Kral K (1999) Motion detection in insect orientation and navigation. Vision Res 39:2749-2766.

Stach S, Giurfa M (2005) The influence of training length on generalization of visual feature assemblies in honeybees. Behav Brain Res 161:8-17.

Tang S, Guo A (2001) Choice behavior of Drosophila facing contradictory visual cues. Science 294:1543-1547.

Tang S, Wolf R, Xu S, Heisenberg M (2004) Visual pattern recognition in Drosophila is invariant for retinal position. Science 305:1020-1022.

Wolf R, Heisenberg M (1991) Basic organization of operant behavior as revealed in Drosophila flight orientation. J Comp Physiol A Neuroethol Sens Neural Behav Physiol 169:699-705.

Wolf R, Wittig T, Liu L, Wustmann G, Eyding D, Heisenberg M (1998) Drosophila mushroom bodies are dispensable for visual, tactile, and motor learning. Learn Mem 5:166-178.

Zars T (2000) Behavioral functions of the insect mushroom bodies. Curr Opin Neurobiol 10:790-795.

Zhang SW, Srinivasan MV (1994) Prior experience enhances pattern discrimination in insect vision. Nature 368:330-332. 